\title{
Prostate-Specific Membrane Antigen-Targeted Radiohalogenated PET and Therapeutic Agents for Prostate Cancer
}

\author{
Steven P. Rowe ${ }^{1}$, Alexander Drzezga ${ }^{2}$, Bernd Neumaier $^{3}$, Markus Dietlein ${ }^{2}$, Michael A. Gorin ${ }^{4}$, Michael R. Zalutsky ${ }^{5}$, \\ and Martin G. Pomper ${ }^{1}$ \\ ${ }^{I}$ The Russell H. Morgan Department of Radiology and Radiological Science, Johns Hopkins University School of Medicine, \\ Baltimore, Maryland; '2Department of Nuclear Medicine, University Hospital of Cologne, Cologne, Germany; ${ }^{3}$ Institute of \\ Radiochemistry and Experimental Molecular Imaging, University Hospital of Cologne, Cologne, Germany; ${ }^{4}$ The James Buchanan \\ Brady Urological Institute and Department of Urology, Johns Hopkins University School of Medicine, Baltimore, Maryland; and \\ ${ }^{5}$ Department of Radiology, Duke University Medical Center, Durham, North Carolina
}

Radiohalogenated agents are often the first line of pursuit in the development of new radiopharmaceuticals-whether antibodies, peptides, or small molecules-because of their ease of synthesis, lack of substantial steric perturbation of the original affinity agent (in some cases, providing enhanced affinity), and capacity to be transformed into therapeutics (in some cases, with a mere switch of an isotope). They often provide proof of a principle before optimization for pharmacokinetics or generation of radiometallated agents, when the latter are necessary. In particular, ${ }^{18} \mathrm{~F}$ has been well integrated into normal clinical work flow in the form of ${ }^{18} \mathrm{~F}-\mathrm{FDG}$ for oncologic imaging, with reliable daily production and distribution to sites for immediate use, without the need for on-site preparation. Here we discuss radiohalogenated versions of imaging and therapeutic agents targeting the prostate-specific membrane antigen (PSMA); these were among the first such agents to be synthesized and used clinically. PSMA is highly expressed on prostate cancer epithelial cells and is currently being extensively investigated around the world as a target for imaging and therapy of prostate cancer. Additionally, the presence of PSMA on nonprostate tumor neovasculature has opened the possibility of PSMA-targeted molecules as generalizable cancer imaging and therapy agents. We focus on ${ }^{18} \mathrm{~F}$-labeled agents for PET, as they begin to redefine-along with the corresponding ${ }^{68} \mathrm{Ga}$-labeled agents discussed elsewhere in this supplement to The Journal of Nuclear Medicine-the management of prostate cancer across a variety of clinical contexts.

Key Words: prostate-specific membrane antigen; PET; ${ }^{18} \mathrm{~F} ;{ }^{211} \mathrm{At}$; biochemical recurrence

J Nucl Med 2016; 57:90S-96S

DOI: 10.2967/jnumed.115.170175

$\mathbf{P}$ rostate cancer $(\mathrm{PC})$ is the most common noncutaneous malignancy in men (1). Initial suspicion often results from elevated levels of prostate-specific antigen (PSA) in serum. The workup at

Received Mar. 1, 2016; revision accepted Aug. 8, 2016

For correspondence or reprints contact: Martin G. Pomper, Johns Hopkins University School of Medicine, Cancer Research Bldg. 2, Room 492, 1550 Orleans St., Baltimore, MD 21231.

E-mail: mpomper@jhmi.edu

COPYRIGHT (C) 2016 by the Society of Nuclear Medicine and Molecular Imaging, Inc. diagnosis involves physical examination and often proceeds to biopsy to validate the presence of cancer. For further staging, restaging, and diagnosis of recurrence, as well as for therapeutic monitoring, noninvasive imaging tests may be incorporated. The most widely accepted imaging modalities used include MRI for the evaluation of patients who have clinically localized primary disease (2) or who are thought to harbor recurrent PC limited to the pelvis (3), contrast-enhanced CT, and ${ }^{99 \mathrm{~m}} \mathrm{Tc}-m e t h y l e n e ~ d i-$ phosphonate bone scanning for the evaluation of metastatic disease in primary and recurrent PC (4). Additionally, in Europe, PET imaging with ${ }^{11} \mathrm{C}$-choline or ${ }^{18} \mathrm{~F}$-fluorocholine has recently gained acceptance for the workup of recurrent PC.

However, those modalities possess significant limitations $(5,6)$. Conventional imaging has not adequately addressed a variety of important clinical issues, including pretherapy risk stratification, reliable pretherapy staging of patients at risk for pelvic lymph node metastases or systemic disease, and the detection of biochemical recurrence at low PSA levels in patients previously treated for PC with curative intent. In the context of such limitations, several agents for the molecular imaging of PC have been developed. These include radiotracers that target lipid metabolism (7), function as nucleoside analogs (8), and bind with high affinity to PC-specific molecular targets, such as the androgen receptor (9), bombesin receptor $(10,11)$, and prostate-specific membrane antigen (PSMA) $(12,13)$. PSMA is a particularly promising target in light of its nearly universal expression in PC and the correlation of increased PSMA expression with tumor aggressiveness (14-16). These various molecular agents may provide the improved imaging reliability necessary to address the shortcomings of conventional imaging currently used to guide PC therapy.

The first PSMA-targeted imaging agent to gain transient clinical acceptance was ${ }^{111}$ In-capromab pendetide (ProstaScint; Aytu BioScience, Inc.), a monoclonal antibody against an intracellular epitope of PSMA (17). Ultimately, the targeting of an intracellular epitope combined with the limitations in spatial resolution and quantification that are inherent to single-photon-emitting radionuclides prevented the acquisition of suitably high-quality images. However, PSMA remained a promising target, and more recent radiolabeled monoclonal antibodies against an extracellular PSMA epitope $(18,19)$ as well as single-photon-emitting $(20,21)$ and positron-emitting (22-25) small-molecule inhibitors of PSMA have been extensively explored. Results continue to suggest that 
PSMA-targeted imaging of $\mathrm{PC}$ is a promising alternative to conventional imaging.

The preliminary success of $\left[{ }^{177} \mathrm{Lu}-\mathrm{DOTA}_{0}, \mathrm{Tyr}_{3}\right]$ octreotate $(26)$ as a therapeutic agent provided a strong rationale for extending smallmolecule, targeted radiopharmaceutical therapy (RPT) to PC. As discussed elsewhere in this supplement, RPT with PSMA-targeted agents is beginning to proliferate in Europe, along with application of the $\beta$-particle emitter ${ }^{177} \mathrm{Lu}(27,28)$. Isotopic analogs of some of the radiohalogenated imaging agents mentioned earlier may also be used for therapy. Accordingly, radiohalogenated agents targeting PSMA may serve as effective theranostic agents in the future.

Here we review the available literature on the preclinical and clinical development of radiohalogenated small-molecule inhibitors of PSMA, with particular emphasis on ${ }^{18} \mathrm{~F}$-labeled agents applicable to PET. We also briefly review the potential of $\alpha$-particle-emitting and Auger electron-emitting radiohalogenated agents for RPT.

\section{RADIOHALOGENATED INHIBITORS OF PSMA AS IMAGING AGENTS}

Several scaffolds have been used in the synthesis of smallmolecule PSMA inhibitors; these include thiols, carbamates, phosphoramidates, phosphinic acids, and ureas (29-33) — the latter two structural categories having been administered to human subjects. A urea-based molecule $\left({ }^{11} \mathrm{C}-\mathrm{MCG}\right.$ or $\left.{ }^{11} \mathrm{C}-\mathrm{DCMC}\right)$ was the first reported preclinical PET agent for PSMA-targeted imaging (Fig. 1) (34). Soon after, the first radiohalogenated small-molecule inhibitor of PSMA ( ${ }^{125}$ I-DCIT) was reported (Table 1; Fig. 1) (35). Both of those early examples of PSMA-targeted small molecules demonstrated uptake in PSMA-expressing PC xenografts derived from a lymph node metastasis, but neither was labeled with an ideal radionuclide for human applications.

Although neither ${ }^{11} \mathrm{C}$-DCMC nor ${ }^{125}$ I-DCIT was investigated in human trials, each showed that in vivo imaging of PSMA was possible in preclinical models. Later-generation radioiodinated agents developed for SPECT and PET (124I) as well as for $\beta$-particle therapy were used in clinical trials $(20,36,37)$. A radiofluorinated smallmolecule inhibitor $\left({ }^{18} \mathrm{~F}-\mathrm{DCFBC}\right)$ (Table 1 ; Fig. 1) was synthesized and found to accumulate in PSMA-positive PC xenografts (38), suggesting an ${ }^{18} \mathrm{~F}$-labeled derivative for translation. As discussed in greater detail later in this review, several considerations are important in choosing a specific radionuclide for PET imaging; ${ }^{18} \mathrm{~F}$ is the superior choice for imaging today in the United States. Although

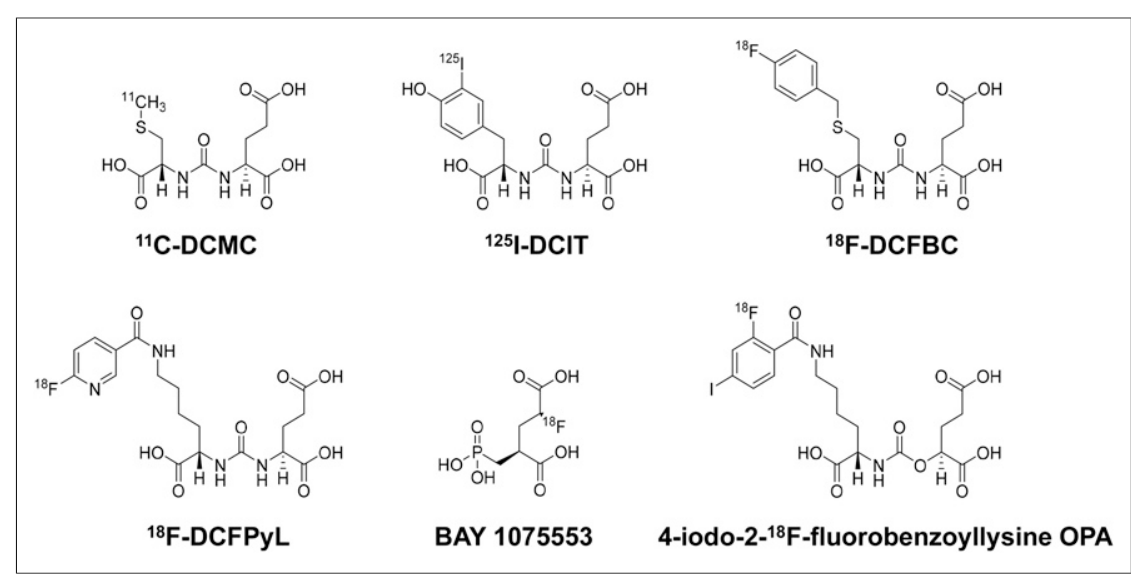

FIGURE 1. Chemical structures of small-molecule inhibitors of PSMA. much of the early clinical literature on PSMA-targeted imaging with small molecules focused on agents labeled with the radiometal ${ }^{68} \mathrm{Ga}$ $(24,39)$, a series of small but informative prospective studies have now been performed with ${ }^{18} \mathrm{~F}-\mathrm{DCFBC}(22,40,41)$. A higher-affinity and more easily synthesized ${ }^{18} \mathrm{~F}$-labeled small molecule for PET imaging of PSMA ( $\left.{ }^{18} \mathrm{~F}-\mathrm{DCFPyL}\right)$ (Table 1; Fig. 1) (42) was subsequently tested in preclinical models and found to have higher uptake than ${ }^{18} \mathrm{~F}-\mathrm{DCFBC}$ in PSMA-expressing PC xenografts (38).

Additional ${ }^{18} \mathrm{~F}$-labeled small molecules that do not contain a urea moiety have also been reported. For example, the phosphonomethylbased small molecule BAY 1075553 (Table 1; Fig. 1) was investigated in both preclinical models (43) and a first-in-man trial that showed safety and efficacy (44).

Recently, Yang et al. reported preclinical results obtained with a series of carbamates that shared structural similarities with the urea-based PSMA inhibitors (30). Those agents, including 4-bromo-2- ${ }^{18} \mathrm{~F}$-fluorobenzoyllysineoxypentanedioic acid (4-bromo-2- ${ }^{18} \mathrm{~F}$-fluorobenzoyllysine OPA) and 4-iodo${ }^{2-18} \mathrm{~F}$-fluorobenzoyllysineoxypentanedioic acid (4-iodo-2- ${ }^{18} \mathrm{~F}$ fluorobenzoyllysine OPA) (Table 1; Fig. 1), showed high tumor uptake and low nontarget tissue retention in preclinical studies. These results suggested that the use of carbamates may be an approach for mitigating toxicity in endoradiotherapeutic applications.

\section{PSMA-TARGETED IMAGING: IMPLICATIONS FOR MANAGING PC}

The potential applications of PSMA-targeted imaging of PC are manifold. PC is often initially diagnosed because of elevated levels of PSA in serum. However, histologic proof of a tumor by biopsy is required for definitive diagnosis (45). Not infrequently, biopsy does not succeed in demonstrating a tumor on the first attempt and may need to be repeated, placing the patient at risk for complications (46). Furthermore, therapeutic decisions, ranging from active surveillance to systemic therapy, are made on the basis of the grade and stage of a tumor (45). However, the performance of imaging procedures currently in use, such as MRI, CT, and bone scanning, has not been satisfactory with regard to the staging of disease $(5,6)$. This issue may lead to a significant proportion of patients being either under- or overtreated. Furthermore, biochemical (PSA) relapse is observed in about $30 \%$ of patients undergoing treatment with curative intent (47). In cases of biochemical recurrence after radical prostatectomy, salvage radiation therapy of the prostatic fossa is often recommended (45) and performed without proof of the location of recurrent tumor tissue exclusively within the intended field of radiation.

The accumulation of clinical data obtained with PSMA-targeted PET imaging agents has suggested that some of the limitations of conventional imaging may be addressed by this new modality. Such an approach may allow for more individually tailored therapy with regard to selective surgery or radiation therapy or for the selection of patients for new therapeutic options (such as PSMA-based RPT (28)). A growing number of studies, mainly with ${ }^{68} \mathrm{Ga}$-labeled PSMA radiotracers, have provided promising results with regard to the 
TABLE 1

Selected Characteristics of Radiohalogenated PSMA-Targeted Agents

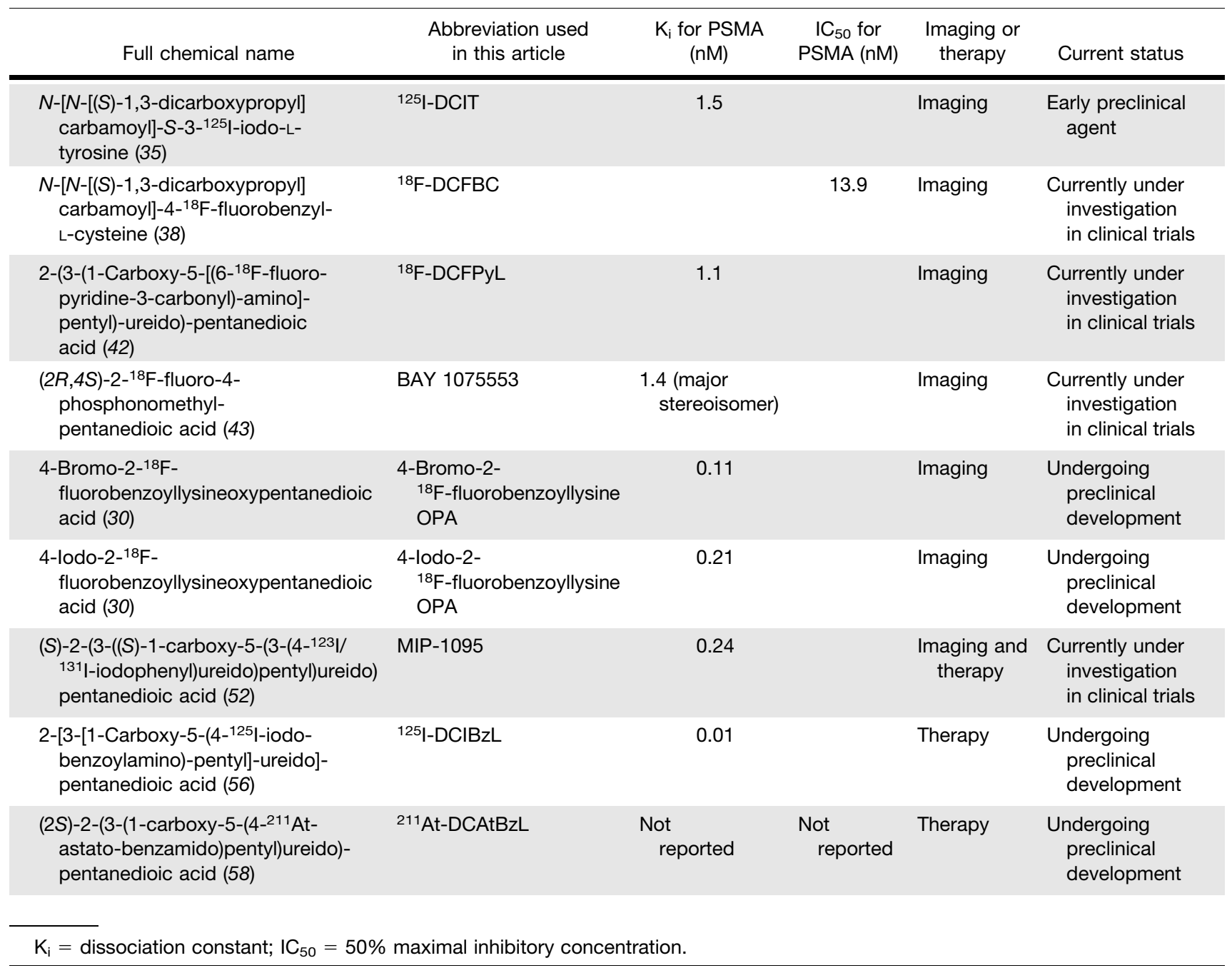

clinical utility of PSMA-targeted imaging $(24,39,48)$. In Europe, ${ }^{68}$ Ga-PSMA PET imaging has already been integrated into the routine diagnostic workup of $\mathrm{PC}$ at selected centers. The rising demand for PSMA-targeted imaging justifies the implementation of ${ }^{18} \mathrm{~F}$-labeled radiotracers for such imaging. Practical considerations regarding ${ }^{18} \mathrm{~F}$ versus ${ }^{68} \mathrm{Ga}$ are discussed later in this review $(22,23,44,49)$.

The first-in-man experience with ${ }^{18} \mathrm{~F}-\mathrm{DCFBC}$ was described by Cho et al. (22). The biodistribution of the radiotracer in human subjects included high uptake in the kidneys. The primary route of excretion of ${ }^{18} \mathrm{~F}$-DCFBC was renal, with the bladder wall having a higher absorbed dose than any other organ. One potential limitation that was apparent with ${ }^{18} \mathrm{~F}$-DCFBC was the persistently high blood-pool activity even $2 \mathrm{~h}$ after radiotracer administration. Nonetheless, ${ }^{18} \mathrm{~F}$-DCFBC uptake was seen in all sites of lymph node and bone metastatic PC that were apparent on conventional imaging with CT and bone scanning as well as in multiple sites that were suspected of having early metastatic disease but that were occult on other types of imaging. Those results implied that PSMA-targeted imaging could have an improved ability to stage patients before local therapy and might also yield more satisfactory identification of sites of disease in patients with biochemical recurrence.

A follow-up prospective study investigating the utility of ${ }^{18} \mathrm{~F}-\mathrm{DCFBC}$ in primary PC was recently published (40). In that study, 13 patients were imaged with ${ }^{18} \mathrm{~F}$-DCFBC PET/CT, and 12 also underwent prostate MRI before radical prostatectomy. Although MRI appeared to be more sensitive for the detection of PC in this small series, ${ }^{18} \mathrm{~F}$-DCFBC PET/CT had a high specificity for the identification of aggressive $\mathrm{PC}$ and also showed no evidence of uptake in benign prostatic hyperplasia. A positive correlation between the Gleason score and ${ }^{18} \mathrm{~F}-\mathrm{DCFBC}$ uptake was found, although the small sample size made it difficult to ascertain the strength of the correlation and the authors reported a wide confidence interval associated with this finding $(\rho, 0.65 ; 95 \%$ confidence interval, $0.16-0.89)$. If such a correlation could be confirmed in larger prospective trials, then PSMA PET might prove to be a noninvasive means of risk stratification for patients with PC.

In a separate prospective trial of 17 patients with known progressive, metastatic PC, Rowe et al. reported that ${ }^{18} \mathrm{~F}-\mathrm{DCFBC}$ $\mathrm{PET} / \mathrm{CT}$ was more sensitive than the conventional imaging modalities 


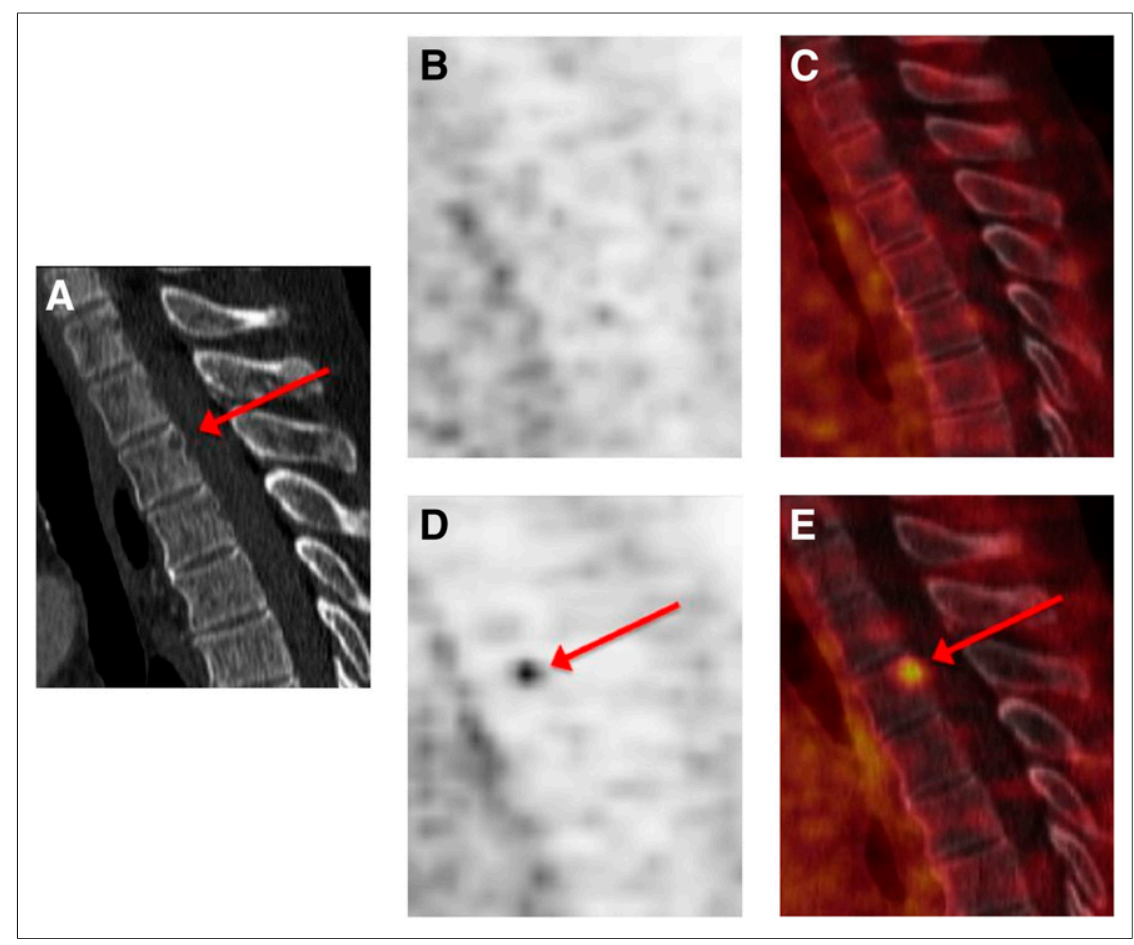

FIGURE 2. Predominantly lytic bone metastasis (arrow) (A) that was occult on ${ }^{68} \mathrm{Ga}-\mathrm{PSMA} P \mathrm{PET}$ (B) and PET/CT (C) but readily apparent on ${ }^{18} \mathrm{~F}-\mathrm{DCFPyL}$ PET (D) and PET/CT (E).

of CT and bone scanning for the detection of suspect lesions (592 positive lesions, along with an additional 63 equivocal lesions, with ${ }^{18} \mathrm{~F}-\mathrm{DCFBC} \mathrm{PET} / \mathrm{CT}$; 520 positive lesions, along with 61 equivocal lesions, with CT and bone scanning) (41). The superior detection by ${ }^{18} \mathrm{~F}-\mathrm{DCFBC}$ was found in patients with both hormonenaïve and castration-resistant PC and was seen with lymph node, bone, and visceral lesions. Again, these findings suggested the potential utility of PSMA-targeted imaging for staging before therapy and for the detection of biochemically recurrent PC.

Chen et al. published promising preclinical data on ${ }^{18} \mathrm{~F}-\mathrm{DCFPyL}$, a PSMA-selective ligand with high binding affinity (42). More recently, Szabo et al. published first-in-man data demonstrating that this radiotracer was safe and showed the expected biodistribution (23). Dietlein et al. reported the first clinical experience in a series of patients examined with both ${ }^{18} \mathrm{~F}-\mathrm{DCFPyL}$ PET/CT and ${ }^{68} \mathrm{Ga}$-PSMA PET/CT (49). In that study, the number and the $\mathrm{SUV}_{\text {max }}$ of PSMA-positive lesions detected by the radiotracers were compared in 14 patients with recurrent PC. In addition to detecting lesions identified by ${ }^{68} \mathrm{Ga}-\mathrm{PSMA},{ }^{18} \mathrm{~F}-\mathrm{DCFPyL}$ PET/CT imaging detected additional lesions in 3 patients (Fig. 2). The authors concluded that ${ }^{18} \mathrm{~F}$-DCFPyL PET/CT provides high image quality and compares favorably with ${ }^{68} \mathrm{Ga}$-PSMA PET/CT, particularly with regard to the sensitive detection of small lesions.

Since the publication of the study of Dietlein et al. (49), ${ }^{18} \mathrm{~F}-\mathrm{DCFPyL}$ imaging has been systematically offered for clinical purposes by the same group. To date, 139 patients have been examined with ${ }^{18} \mathrm{~F}$-DCFPyL for a variety of diagnostic purposes; however, patients were referred predominantly for localization after biochemical recurrence (unpublished data). Other indications have included staging at primary diagnosis, restaging, therapy planning and disease monitoring in cases of castration resistance, and selection for ${ }^{177} \mathrm{Lu}$-PSMA RPT. ${ }^{18} \mathrm{~F}$-DCFPyL has proven to be of high value for detecting local recurrences and lymph node and bone metastases, even at very low PSA levels $(<0.5 \mathrm{ng} / \mathrm{dL})$ (Fig. 3). A systematic, retrospective evaluation of the collected clinical ${ }^{18} \mathrm{~F}$-DCFPyL PET/CT data (and a comparison group of ${ }^{68} \mathrm{Ga}$-PSMA data) is under way and will be published elsewhere.

\section{${ }^{18} \mathrm{~F}$ VERSUS ${ }^{68} \mathrm{Ga}$ : PRACTICAL CONSIDERATIONS}

At present, for PSMA-targeted PET imaging, ${ }^{68} \mathrm{Ga}$-labeled radiotracers are most commonly used. A major advantage of ${ }^{68} \mathrm{Ga}$ labeling is the commercial availability of germanium/gallium generators. The availability of such generators allows on-site synthesis of ${ }^{68} \mathrm{Ga}$-labeled PET radiotracers at centers without access to a cyclotron and with only a modest investment in infrastructure dedicated to radiopharmaceutical synthesis. ${ }^{18} \mathrm{~F}$ labeling requires access to a cyclotron but has several distinct advantages. Generally, the higher production capability allows the synthesis of larger batches, allowing many examinations to be performed in sequence on the basis of a single synthesis. A single ${ }^{68} \mathrm{Ga}$-based synthesis will usually enable the production of a sufficient amount of radiotracer for only 3 or 4 examinations. Additionally, the longer physical half-life of ${ }^{18} \mathrm{~F}$ (109 $\left.\mathrm{min}\right)$ than of ${ }^{68} \mathrm{Ga}(68 \mathrm{~min})$ allows for easier transportation of the radiotracer from central production sites to remote imaging centers, obviating the need for on-site radiosynthesis personnel.
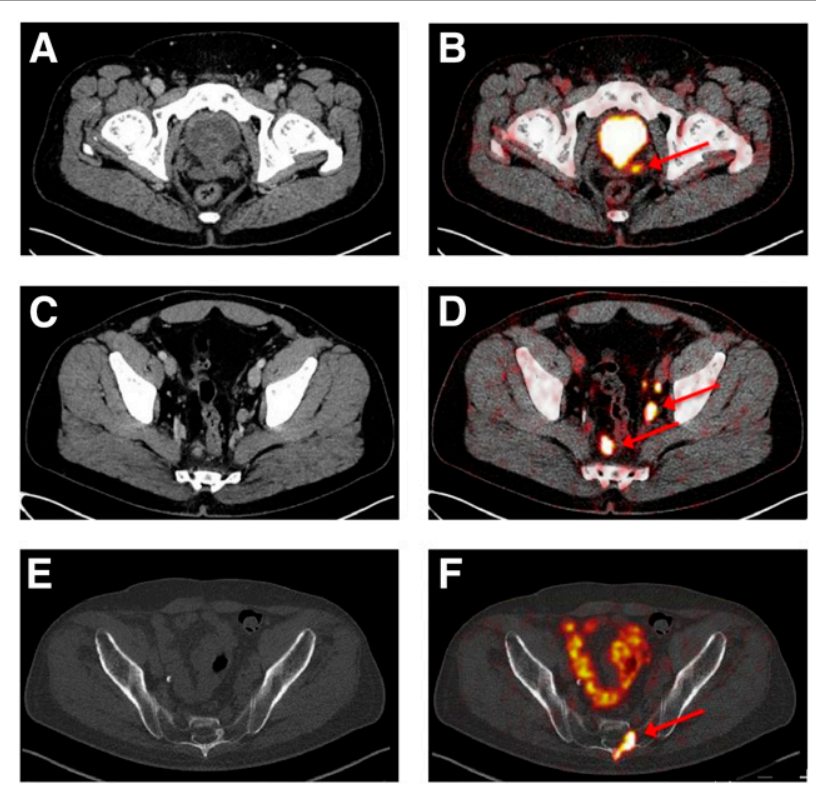

FIGURE 3. Detection of wide variety of PC lesions with ${ }^{18} \mathrm{~F}-\mathrm{DCFPyL}$. Locally recurrent PC (CT $[\mathrm{A}]$ and $\left.{ }^{18} \mathrm{~F}-\mathrm{DCFPyL} \mathrm{PET} / \mathrm{CT}[\mathrm{B}]\right)$ and lymph node (CT [C] and ${ }^{18} \mathrm{~F}$-DCFPyL PET/CT [D]) and bone (CT [E] and ${ }^{18} \mathrm{~F}-$ DCFPyL PET/CT [F]) lesions were visible as marked focal radiotracer uptake (arrows). 
At present, the production of ${ }^{18} \mathrm{~F}-\mathrm{DCFPyL}$ can be performed in 2 steps. The first step consists of the preparation of an activated ester (2,3,5,6-tetrafluorophenyl 6 - ${ }^{18} \mathrm{~F}$-fluoronicotinate), which is subsequently coupled to the $(S)$ Lys-C $(\mathrm{O})-(S)$ Glu motif. The final product, ${ }^{18} \mathrm{~F}$-DCFPyL, is then purified by preparative highperformance liquid chromatography. A purification method based on solid-phase extraction is in development. Translation of this radiosynthesis method to commercially available synthesis modules with disposable cassettes is imminent (personal communication). The expense for the synthesis of ${ }^{18} \mathrm{~F}$-DCFPyL with an automated system is expected to be similar to that for the synthesis of ${ }^{68} \mathrm{Ga}$-PSMA. Consequently, ${ }^{18} \mathrm{~F}-\mathrm{DCFPyL}$ could be produced in larger amounts than its ${ }^{68} \mathrm{Ga}$-labeled analog and at a similar expense.

Differences in image quality may represent another important factor supporting the adoption of ${ }^{18}$ F-labeled PSMA-targeted radiotracers. As a result of favorable dosimetry, the injection of higher radiotracer doses leads to improved image statistics. ${ }^{68} \mathrm{Ga}$ also has a lower positron yield (89.14\%; that for ${ }^{18} \mathrm{~F}$ is $96.86 \%(50)$ ) and exhibits several single-photon emissions, resulting in an increase in image noise. Compensating by increasing the scan time for the injected dose is difficult because of the shorter half-life of ${ }^{68} \mathrm{Ga}$. A further advantage of ${ }^{18} \mathrm{~F}$ is that its spatial resolution is higher than that of ${ }^{68} \mathrm{Ga}$ because of its lower positron energy $\left(1,899 \mathrm{keV}\right.$ for ${ }^{68} \mathrm{Ga}$ vs. $633 \mathrm{keV}$ for ${ }^{18} \mathrm{~F}$ ). In the case of lower kinetic energy, the locations of the positron emission and the annihilation point are closer, resulting in better resolution of PET images. Accordingly, higher intrinsic image resolution for ${ }^{18} \mathrm{~F}$-labeled radiotracers is possible.

\section{RADIOHALOGENATED PSMA-TARGETED SMALL MOLECULES FOR THERAPY}

A key aspect of targeted RPT is selecting a radionuclide with characteristics that balance minimum toxicity to normal tissues and maximum efficacy for a particular clinical application. Because the clinical evaluation of novel cancer therapeutics is generally initiated in patients with advanced disease, PSMA-targeted RPT was first evaluated with $\beta$-emitters whose longer range should have been advantageous in that setting. The targeting potential of a series of radioiodinated PSMA inhibitors was demonstrated by Hillier et al. (51) and formed the basis for the clinical evaluation of ${ }^{131}$ I-MIP-1095 in 28 patients with metastatic castration-resistant PC (37). However, as discussed elsewhere in this supplement, most current work with $\beta$-emitters for PSMA-targeted RPT is focused on radiometals such as ${ }^{177} \mathrm{Lu}$ because of perceived advantages in emission spectra and convenience.

Perhaps the most important potential application of PSMAtargeted RPT is the treatment of micrometastatic disease. In that setting, it is essential to be able to deliver tumoricidal doses of radiation to small clusters of malignant cells while sparing neighboring normal tissues. Two types of radiation-Auger electrons and $\alpha$-particles-have a higher potency and a shorter tissue range than $\beta$-particles; these characteristics should make them suitable for the treatment of microscopic disease (52). The linear energy transfers of Auger electrons and $\alpha$-particles are about 4-26 and $100 \mathrm{keV} / \mu \mathrm{m}$, respectively (that for most $\beta$-emitters is $\sim 0.2 \mathrm{keV} / \mu \mathrm{m}$ ); these values confer a greater deposition of energy within small volumes and a higher cytotoxicity (53). Unlike $\beta$-particles, which have path lengths of up to 1,000 cell diameters, the range of $\alpha$-particles is only about $5-10$ cell diameters and the range of most Auger electrons is considerably smaller than 1 cell. The feasibility of treating metastatic PC with an $\alpha$-emitting radiopharmaceutical was demonstrated in a phase III trial with ${ }^{223} \mathrm{RaCl}_{2}$ (Alpharadin; Bayer) (54); however, its mechanism of uptake limits its application to bone metastases. Exploiting PSMA as a molecular target offers the exciting prospect of treating micrometastatic disease in both bone and soft tissues.

With regard to Auger electron emitters, Kiess et al. evaluated the therapeutic potential of ${ }^{125}$ I-DCIBzL (Table 1; Fig. 4) for treating PC (55). ${ }^{125} \mathrm{I}$ has been the prototypical radionuclide for Auger electron therapy experiments because it emits an average of approximately 20 electrons per decay, with a tissue range of less than $0.5 \mu \mathrm{m}$, and requires localization of the site of its decay to the cell nucleus for maximum cytotoxicity (56). In cell culture experiments, ${ }^{125}$ I-DCIBzL treatment resulted in PSMA-specific killing, as evidenced by increased DNA damage and decreased clonogenic survival in PSMA-positive (PC3 PIP) versus PSMA-negative (PC3 flu) cells. Treatment of PC3 PIP tumors with a 111-MBq dose of ${ }^{125} \mathrm{I}-\mathrm{DCIBzL}$ resulted in a significant tumor growth delay. Although these results are highly encouraging, ${ }^{125}$ I may not be the ideal Auger electron emitter for clinical translation, in part because of its $60-\mathrm{d}$ half-life. Two Auger electron emitters that may offer advantages for PSMA-targeted radiotherapy are radiohalogens- ${ }^{123}$ I (13-h half-life) and ${ }^{77} \mathrm{Br}$ (57-h half-life) — and studies evaluating their suitability with appropriately designed PSMA inhibitors are under way.

The radiohalogen ${ }^{211} \mathrm{At}$ has particularly favorable characteristics for clinical development, including a 7.2-h half-life, $100 \%$ $\alpha$-particle emission per decay, and the absence of $\alpha$-particleemitting daughters, which can lead to excessive radiation doses to normal tissues when released from the parent molecule $(52,53)$. To investigate the therapeutic potential of PSMA-targeted ${ }^{211} \mathrm{At}$, ${ }^{211}$ At-DCAtBzL (Table 1; Fig. 4) was synthesized and evaluated in PSMA-positive PC3 PIP and PSMA-negative PC3 flu human prostate carcinoma models (57). A paired-label biodistribution experiment demonstrated that the uptake of ${ }^{211}$ At-DCAtBzL in the PSMA-positive xenograft was high over a time course relevant to the half-life of ${ }^{211} \mathrm{At}$ and increased with time, reaching a value of $41.5 \pm 18.4$ (mean $\pm \mathrm{SD}$ ) percentage injected dose/g at $18 \mathrm{~h}$. Except at $18 \mathrm{~h}$, the uptake of the coadministered ${ }^{131} \mathrm{I}$ analog in

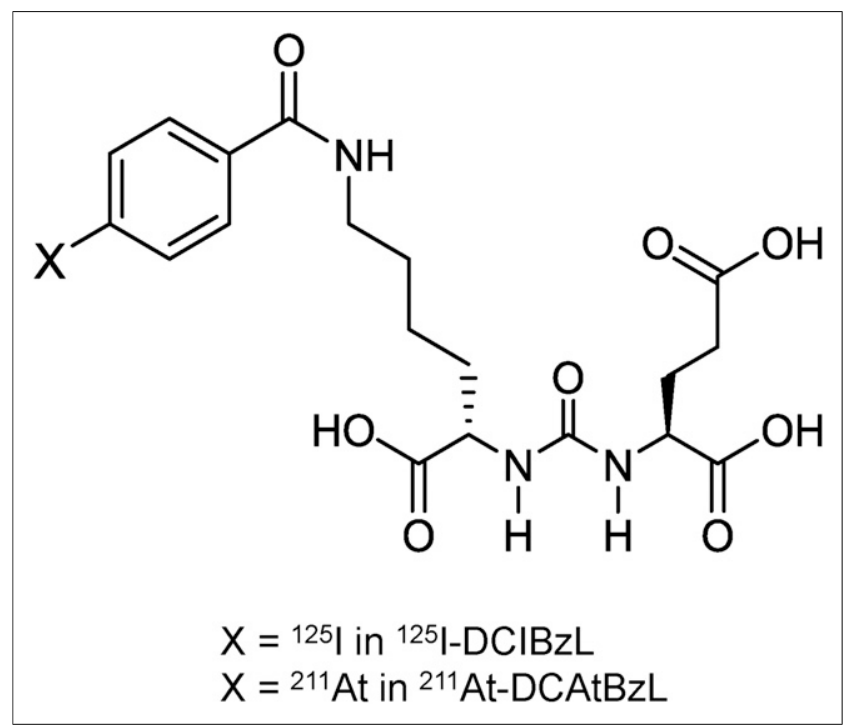

FIGURE 4. Auger electron-emitting ( $\left.{ }^{125} \mathrm{I}-\mathrm{DCIBzL}\right)$ and a-particleemitting $\left({ }^{211} \mathrm{At}-\mathrm{DCAtBzL}\right)$ radiohalogenated small-molecule inhibitors of PSMA. 
the PSMA-positive xenograft was significantly higher, by about $25 \%-33 \%$. That observation, in concert with increased ${ }^{211} \mathrm{At} \mathrm{lev-}$ els in tissues known to accumulate free halides (stomach and thyroid), suggested that some deastatination of ${ }^{211}$ At-DCAtBzL had occurred. Nonetheless, these results are highly encouraging, particularly for a non-protein-based agent, because-to the best of our knowledge - that uptake is the highest level of sustained tumor uptake that has been reported for a ${ }^{211}$ At-labeled targeted radiotherapeutic agent (58).

The therapeutic potential of ${ }^{211}$ At-DCAtBzL was first investigated in subcutaneous xenografts and then in a newly developed micrometastatic PC model (57). Treatment with ${ }^{211}$ At-DCAtBzL significantly improved survival in the micrometastatic disease model; however, parallel long-term toxicity studies demonstrated that a meaningful therapeutic benefit occurred in a dose range that also resulted in delayed renal toxicity. $\alpha$-Camera imaging demonstrated selective uptake of ${ }^{211}$ At in renal proximal tubules, consistent with the presence of PSMA in these tissues (59). Although ${ }^{211}$ At-DCAtBzL is not suitable for clinical translation, this compound provided valuable confirmation that ${ }^{211}$ At-labeled PSMA inhibitors with intact tumor-localizing capacity and reasonable in vivo stability can be synthesized.

\section{FUTURE DIRECTIONS FOR PSMA-TARGETED MANAGEMENT OF PC}

The rapid adoption in Europe of predominantly ${ }^{68} \mathrm{Ga}$-labeled compounds for diagnostic imaging and ${ }^{177} \mathrm{Lu}$-labeled compounds for RPT hints at the possible transformative capacity of these agents. However, the vast majority of the literature on clinical PSMA-targeted imaging and therapy is retrospective, with only a few small prospective studies to date $(40,41,60)$. The most pressing need in the field of PSMA-based management of PC is a commitment to large, multicenter, prospective, well-designed clinical trials that can definitively prove the utility of these agents in specific clinical settings. Further study of PSMA as an imaging biomarker and noninvasive means of determining levels of androgen signaling during therapy and judging the radiographic response to therapy are also of broad interest and should be studied in a prospective, systematic fashion.

In addition to studies evaluating the role of PSMA-targeted imaging agents in the context of defined clinical issues, studies evaluating the global impact on clinical decision making in PC patients should also be done. Such studies might take a form similar to the National Oncologic PET Registry; that is, prescan and postscan evaluations of treatment plans could be used to gauge the impact of imaging findings on patient management.

Increasing numbers of case reports and small series are demonstrating the possible utility of PSMA-targeted imaging in nonprostate cancers. The localization of a radiotracer in those cancers appears to be based on PSMA expression in tumor neovasculature (61). Clear cell renal cell carcinoma (62-64) and cancers of the thyroid (65), breast (66), and colon (67) are among the tumor types reported to have PSMA-targeted radiotracer uptake. With thoughtful, prospective trials to define the clinical utility of PSMA-targeted imaging in these cancers, PSMA-targeted imaging and therapy may prove to have broad implications in the field of oncology. Indeed, diseases such as metastatic clear cell renal cell carcinoma often have a less indolent course than PC, suggesting that the impact of PSMA-targeted imaging may actually be greater in those conditions.

\section{CONCLUSION}

With the exquisite specificity and high sensitivity afforded by PSMA PET radiotracers, the hope is that clinicians will increasingly incorporate PSMA-targeted imaging as part of the routine evaluation and management of men with PC. The use of radiohalogensalready proving useful for therapy in the context of recent clinical trials incorporating ${ }^{131} \mathrm{I}$-is being extended to $\alpha$-particle emitters, such as ${ }^{211} \mathrm{At}$, with an attendant focus on micrometastatic disease.

\section{DISCLOSURE}

This work was supported in part by NIH CA124675, CA184228, and CA183031. Under a licensing agreement between Progenics and Johns Hopkins University, Martin G. Pomper is entitled to royalties on an invention described in this article. This arrangement has been reviewed and approved by Johns Hopkins University in accordance with its conflict-of-interest policies. No other potential conflict of interest relevant to this article was reported.

\section{REFERENCES}

1. Torre LA, Bray F, Siegel RL, Ferlay J, Lortet-Tieulent J, Jemal A. Global cancer statistics, 2012. CA Cancer J Clin. 2015;65:87-108.

2. Weinreb JC, Barentsz JO, Choyke PL, et al. PI-RADS Prostate ImagingReporting and Data System: 2015, Version 2. Eur Urol. 2016;69:16-40.

3. Silverman JM, Krebs TL. MR imaging evaluation with a transrectal surface coil of local recurrence of prostatic cancer in men who have undergone radical prostatectomy. AJR. 1997;168:379-385.

4. Hricak H, Schoder H, Pucar D, et al. Advances in imaging in the postoperative patient with a rising prostate-specific antigen level. Semin Oncol. 2003;30:616-634.

5. Kane CJ, Amling CL, Johnstone PA, et al. Limited value of bone scintigraphy and computed tomography in assessing biochemical failure after radical prostatectomy. Urology. 2003;61:607-611.

6. Le JD, Tan N, Shkolyar E, et al. Multifocality and prostate cancer detection by multiparametric magnetic resonance imaging: correlation with whole-mount histopathology. Eur Urol. 2015;67:569-576.

7. Bauman G, Belhocine T, Kovacs M, Ward A, Beheshti M, Rachinsky I. ${ }^{18}$ Ffluorocholine for prostate cancer imaging: a systematic review of the literature. Prostate Cancer Prostatic Dis. 2012;15:45-55.

8. Sun H, Sloan A, Mangner TJ, et al. Imaging DNA synthesis with $\left[{ }^{18} \mathrm{~F}\right] \mathrm{FMAU}$ and positron emission tomography in patients with cancer. Eur J Nucl Med Mol Imaging. 2005;32:15-22.

9. Beattie BJ, Smith-Jones PM, Jhanwar YS, et al. Pharmacokinetic assessment of the uptake of $16 \beta{ }^{18} \mathrm{~F}$-fluoro-5 $\alpha$-dihydrotestosterone (FDHT) in prostate tumors as measured by PET. J Nucl Med. 2010;51:183-192.

10. Lears KA, Ferdani R, Liang K, et al. In vitro and in vivo evaluation of ${ }^{64} \mathrm{Cu}-$ labeled SarAr-bombesin analogs in gastrin-releasing peptide receptor-expressing prostate cancer. J Nucl Med. 2011;52:470-477.

11. Schwarzenböck SM, Schmeja P, Kurth J, et al. Comparison of $\left[{ }^{11} \mathrm{C}\right]$ choline $\left(\left[{ }^{11} \mathrm{C}\right] \mathrm{CHO}\right)$ and $\left[{ }^{18} \mathrm{~F}\right]$ bombesin (BAY 86-4367) as imaging probes for prostate cancer in a PC-3 prostate cancer xenograft model. Mol Imaging Biol. 2016;18:393-401.

12. Kiess AP, Cho SY, Pomper MG. Translational molecular imaging of prostate cancer. Curr Radiol Rep. 2013;1:216-226.

13. Maurer T, Eiber M, Schwaiger M, Gschwend JE. Current use of PSMA-PET in prostate cancer management. Nat Rev Urol. 2016;13:226-235.

14. Sweat SD, Pacelli A, Murphy GP, Bostwick DG. Prostate-specific membrane antigen expression is greatest in prostate adenocarcinoma and lymph node metastases. Urology. 1998;52:637-640.

15. Perner S, Hofer MD, Kim R, et al. Prostate-specific membrane antigen expression as a predictor of prostate cancer progression. Hum Pathol. 2007;38:696-701.

16. Ross JS, Sheehan CE, Fisher HA, et al. Correlation of primary tumor prostatespecific membrane antigen expression with disease recurrence in prostate cancer. Clin Cancer Res. 2003;9:6357-6362.

17. Manyak MJ. Indium-111 capromab pendetide in the management of recurrent prostate cancer. Expert Rev Anticancer Ther. 2008;8:175-181.

18. Osborne JR, Green DA, Spratt DE, et al. A prospective pilot study of ${ }^{89} \mathrm{Zr}-\mathrm{J} 591 /$ prostate specific membrane antigen positron emission tomography in men with localized prostate cancer undergoing radical prostatectomy. J Urol. 2014;191: 1439-1445. 
19. Pandit-Taskar N, O’Donoghue JA, Durack JC, et al. A phase I/II study for analytic validation of ${ }^{89} \mathrm{Zr}$-J591 immunoPET as a molecular imaging agent for metastatic prostate cancer. Clin Cancer Res. 2015;21:5277-5285.

20. Barrett JA, Coleman RE, Goldsmith SJ, et al. First-in-man evaluation of 2 highaffinity PSMA-avid small molecules for imaging prostate cancer. J Nucl Med. 2013;54:380-387.

21. Hillier SM, Maresca KP, Lu G, et al. ${ }^{99 \mathrm{~m} T c-l a b e l e d ~ s m a l l-m o l e c u l e ~ i n h i b i t o r s ~ o f ~}$ prostate-specific membrane antigen for molecular imaging of prostate cancer. $J$ Nucl Med. 2013;54:1369-1376.

22. Cho SY, Gage KL, Mease RC, et al. Biodistribution, tumor detection, and radiation dosimetry of ${ }^{18} \mathrm{~F}$-DCFBC, a low-molecular-weight inhibitor of prostatespecific membrane antigen, in patients with metastatic prostate cancer. $\mathrm{J} \mathrm{Nucl}$ Med. 2012;53:1883-1891.

23. Szabo Z, Mena E, Rowe SP, et al. Initial evaluation of $\left[{ }^{18} \mathrm{~F}\right] \mathrm{DCFPyL}$ for prostatespecific membrane antigen (PSMA)-targeted PET imaging of prostate cancer. Mol Imaging Biol. 2015;17:565-574.

24. Afshar-Oromieh A, Avtzi E, Giesel FL, et al. The diagnostic value of PET/CT imaging with the ${ }^{68}$ Ga-labelled PSMA ligand HBED-CC in the diagnosis of recurrent prostate cancer. Eur J Nucl Med Mol Imaging. 2015;42:197-209.

25. Maurer T, Gschwend JE, Rauscher I, et al. Diagnostic efficacy of ${ }^{68}$ galliumPSMA positron emission tomography compared to conventional imaging for lymph node staging of 130 consecutive patients with intermediate to high risk prostate cancer. J Urol. 2016;195:1436-1443.

26. Halperin DM, Dasari A, Yao JC. $\left[{ }^{177} \mathrm{Lu}-\mathrm{DOTA}_{0}, \mathrm{Tyr}_{3}\right]$-octreotate in the treatment of midgut neuroendocrine tumors. Future Oncol. 2016;12:313-321.

27. Weineisen M, Schottelius M, Simecek J, et al. ${ }^{68} \mathrm{Ga}$ - and ${ }^{177} \mathrm{Lu}$-labeled PSMA I\&T: optimization of a PSMA-targeted theranostic concept and first proof-ofconcept human studies. J Nucl Med. 2015;56:1169-1176.

28. Baum RP, Kulkarni HR, Schuchardt C, et al. ${ }^{177}$ Lu-labeled prostate-specific membrane antigen radioligand therapy of metastatic castration-resistant prostate cancer: safety and efficacy. J Nucl Med. 2016;57:1006-1013.

29. Zhou J, Neale JH, Pomper MG, Kozikowski AP. NAAG peptidase inhibitors and their potential for diagnosis and therapy. Nat Rev Drug Discov. 2005;4:1015-1026.

30. Yang X, Mease RC, Pullambhatla M, et al. $\left[{ }^{18} \mathrm{~F}\right]$ fluorobenzoyllysinepentanedioic acid carbamates: new scaffolds for positron emission tomography (PET) imaging of prostate-specific membrane antigen (PSMA). J Med Chem. 2016;59:206-218.

31. Eder M, Eisenhut M, Babich J, Haberkorn U. PSMA as a target for radiolabelled small molecules. Eur J Nucl Med Mol Imaging. 2013;40:819-823.

32. Machulkin AE, Ivanenkov YA, Aladinskaya AV, et al. Small-molecule PSMA ligands: current state, SAR and perspectives. J Drug Target. 2016;24:679-693.

33. Kiess AP, Banerjee SR, Mease RC, et al. Prostate-specific membrane antigen as a target for cancer imaging and therapy. Q J Nucl Med Mol Imaging. 2015;59:241-268.

34. Pomper MG, Musachio JL, Zhang J, et al. ${ }^{11} \mathrm{C}-\mathrm{MCG}$ : synthesis, uptake selectivity, and primate PET of a probe for glutamate carboxypeptidase II (NAALADase). Mol Imaging. 2002;1:96-101.

35. Foss CA, Mease RC, Fan H, et al. Radiolabeled small-molecule ligands for prostate-specific membrane antigen: in vivo imaging in experimental models of prostate cancer. Clin Cancer Res. 2005;11:4022-4028.

36. Chen Y, Foss CA, Byun Y, et al. Radiohalogenated prostate-specific membrane antigen (PSMA)-based ureas as imaging agents for prostate cancer. J Med Chem. 2008;51:7933-7943.

37. Zechmann CM, Afshar-Oromieh A, Armor T, et al. Radiation dosimetry and first therapy results with a ${ }^{124} \mathrm{I} /{ }^{131} \mathrm{I}$-labeled small molecule (MIP-1095) targeting PSMA for prostate cancer therapy. Eur J Nucl Med Mol Imaging. 2014;41:1280-1292.

38. Mease RC, Dusich CL, Foss CA, et al. $N-[N-[(S)-1,3$-dicarboxypropyl $]$ carbamoyl]-4-[ $\left.{ }^{18} \mathrm{~F}\right]$ fluorobenzyl-L-cysteine, $\left[{ }^{18} \mathrm{~F}\right] \mathrm{DCFBC}$ : a new imaging probe for prostate cancer. Clin Cancer Res. 2008;14:3036-3043.

39. Eiber M, Maurer T, Souvatzoglou M, et al. Evaluation of hybrid ${ }^{68}$ Ga-PSMA ligand PET/CT in 248 patients with biochemical recurrence after radical prostatectomy. J Nucl Med. 2015;56:668-674.

40. Rowe SP, Gage KL, Faraj SF, et al. ${ }^{18} \mathrm{~F}-\mathrm{DCFBC}$ PET/CT for PSMA-based detection and characterization of primary prostate cancer. J Nucl Med. 2015; 56:1003-1010.

41. Rowe SP, Macura KJ, Ciarallo A, et al. Comparison of prostate-specific membrane antigen-based ${ }^{18} \mathrm{~F}$-DCFBC PET/CT to conventional imaging modalities for detection of hormone-naïve and castration-resistant metastatic prostate cancer. J Nucl Med. 2016;57:46-53.

42. Chen Y, Pullambhatla M, Foss CA, et al. 2-(3-\{1-Carboxy-5-[(6- $\left[{ }^{18} \mathrm{~F}\right]$ fluoropyridine-3-carbonyl)-amino]-pentyl $\}$-ureido)-pentanedioic acid, $\left[{ }^{18} \mathrm{~F}\right] \mathrm{DCFPyL}$, a PSMA-based PET imaging agent for prostate cancer. Clin Cancer Res. 2011; 17:7645-7653

43. Lesche R, Kettschau G, Gromov AV, et al. Preclinical evaluation of BAY 1075553, a novel ${ }^{18} \mathrm{~F}$-labelled inhibitor of prostate-specific membrane antigen for PET imaging of prostate cancer. Eur J Nucl Med Mol Imaging. 2014;41:89-101.
44. Beheshti M, Kunit T, Haim S, et al. BAY 1075553 PET-CT for staging and restaging prostate cancer patients: comparison with $\left[{ }^{18} \mathrm{~F}\right]$ fluorocholine PET-CT (phase I study). Mol Imaging Biol. 2015;17:424-433.

45. Parker C, Gillessen S, Heidenreich A, Horwich A; ESMO Guidelines Committee. Cancer of the prostate: ESMO clinical practice guidelines for diagnosis, treatment and follow-up. Ann Oncol. 2015;26(suppl 5):v69-v77.

46. Uchio EM, Aslan M, Wells CK, Calderone J, Concato J. Impact of biochemical recurrence in prostate cancer among US veterans. Arch Intern Med. 2010; 170:1390-1395.

47. Chittenden SJ, Hindorf C, Parker CC, et al. A phase 1, open-label study of the biodistribution, pharmacokinetics, and dosimetry of ${ }^{223} \mathrm{Ra}$-dichloride in patients with hormone-refractory prostate cancer and skeletal metastases. J Nucl Med. 2015;56:1304-1309.

48. Maurer T, Weirich G, Schottelius M, et al. Prostate-specific membrane antigenradioguided surgery for metastatic lymph nodes in prostate cancer. Eur Urol. 2015;68:530-534.

49. Dietlein M, Kobe C, Kuhnert G, et al. Comparison of $\left[{ }^{18} \mathrm{~F}\right] \mathrm{DCFPyL}$ and $\left[{ }^{68} \mathrm{Ga}\right]$ Ga-PSMA-HBED-CC for PSMA-PET imaging in patients with relapsed prostate cancer. Mol Imaging Biol. 2015;17:575-584.

50. Sanchez-Crespo A. Comparison of gallium-68 and fluorine-18 imaging characteristics in positron emission tomography. Appl Radiat Isot. 2013;76:55-62.

51. Hillier SM, Maresca KP, Femia FJ, et al. Preclinical evaluation of novel glutamate-urea-lysine analogues that target prostate-specific membrane antigen as molecular imaging pharmaceuticals for prostate cancer. Cancer Res. 2009; 69:6932-6940.

52. Aghevlian S, Boyle AJ, Reilly RM. Radioimmunotherapy of cancer with high linear energy transfer (LET) radiation delivered by radionuclides emitting $\alpha$-particles or Auger electrons. Adv Drug Deliv Rev. December 17, 2015 [Epub ahead of print].

53. Zalutsky MR. Radionuclide therapy. In: Vértes A, Nagy S, Klencsár Z, et al., eds. Handbook of Nuclear Chemistry: Radiochemistry and Radiopharmaceutical Chemistry in Life Sciences. 2nd ed. Vol. 4. Berlin, Germany: Springer; 2011: 2179-2212.

54. Parker C, Heinrich D, O'Sullivan JM, et al. Overall survival benefit of radium223 chloride (Alpharadin ${ }^{\mathrm{TM}}$ ) in the treatment of patients with symptomatic bone metastases in castration-resistant prostate cancer (CRPC): a phase III randomized trial (ALSYMPCA) [abstract]. Eur J Cancer. 2011;47(suppl 2):3.

55. Kiess AP, Minn I, Chen Y, et al. Auger radiopharmaceutical therapy targeting prostate-specific membrane antigen. J Nucl Med. 2015;56:1401-1407.

56. Kassis AI. The amazing world of Auger electrons. Int J Radiat Biol. 2004;80: 789-803.

57. Kiess A, Minn IL, Vaidyanathan G, et al. (2S)-2-(3-(1-carboxy-5-(4- $\left[{ }^{211} \mathrm{At}\right]$ astatobenzamido)pentyl)ureido)-pentanedioic acid for PSMA-targeted $\alpha$-particle radiopharmaceutical therapy. $J$ Nucl Med. May 26, 2016 [Epub ahead of print]

58. Guérard F, Gestin JF, Brechbiel MW. Production of [ $\left.{ }^{211} \mathrm{At}\right]$-astatinated radiopharmaceuticals and applications in targeted $\alpha$-particle therapy. Cancer Biother Radiopharm. 2013;28:1-20.

59. Rovenská M, Hlouchova K, Sacha P, et al. Tissue expression and enzymologic characterization of human prostate specific membrane antigen and its rat and pig orthologs. Prostate. 2008;68:171-182.

60. Morigi JJ, Stricker PD, van Leeuwen PJ, et al. Prospective comparison of ${ }^{18} \mathrm{~F}-$ fluoromethylcholine versus ${ }^{68} \mathrm{Ga}$-PSMA PET/CT in prostate cancer patients who have rising PSA after curative treatment and are being considered for targeted therapy. J Nucl Med. 2015;56:1185-1190.

61. Chang SS, O'Keefe DS, Bacich DJ, Reuter VE, Heston WD, Gaudin PB. Prostate-specific membrane antigen is produced in tumor-associated neovasculature. Clin Cancer Res. 1999;5:2674-2681.

62. Demirci E, Ocak M, Kabasakal L, et al. ${ }^{68} \mathrm{Ga}$-PSMA PET/CT imaging of metastatic clear cell renal cell carcinoma. Eur J Nucl Med Mol Imaging. 2014;41:1461-1462.

63. Rowe SP, Gorin MA, Hammers HJ, et al. Imaging of metastatic clear cell renal cell carcinoma with PSMA-targeted ${ }^{18}$ F-DCFPyL PET/CT. Ann Nucl Med. 2015;29:877-882.

64. Rowe SP, Gorin MA, Hammers HJ, Pomper MG, Allaf ME, Javadi MS. Detection of ${ }^{18}$ F-FDG PET/CT occult lesions with ${ }^{18} \mathrm{~F}$-DCFPyL PET/CT in a patient with metastatic renal cell carcinoma. Clin Nucl Med. 2016;41: 83-85.

65. Verburg FA, Krohn T, Heinzel A, Mottaghy FM, Behrendt FF. First evidence of PSMA expression in differentiated thyroid cancer using $\left[{ }^{68} \mathrm{Ga}\right]$ PSMA-HBED-CC PET/CT. Eur J Nucl Med Mol Imaging. 2015;42:1622-1623.

66. Sathekge M, Modiselle M, Vorster M, et al. ${ }^{68} \mathrm{Ga}$-PSMA imaging of metastatic breast cancer. Eur J Nucl Med Mol Imaging. 2015;42:1482-1483.

67. Huang YT, Fong W, Thomas P. Rectal carcinoma on ${ }^{68}$ Ga-PSMA PET/CT. Clin Nucl Med. 2016;41:e167-e168. 